\title{
Reducing readmissions in patients with cirrhosis: the time to act is now
}

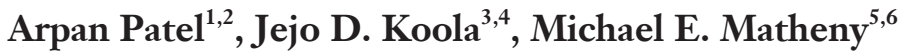 \\ ${ }^{1}$ Vatche and Tamar Manoukian Division of Digestive Diseases, Department of Medicine, David Geffen School of Medicine at UCLA, Los Angeles, \\ CA, USA; ${ }^{2}$ Veterans Affairs Greater Los Angeles Healthcare System, Los Angeles, CA, USA; ${ }^{3}$ UC Health Department of Biomedical Informatics, \\ University of California, San Diego, La Jolla, CA, USA; ${ }^{4}$ Division of Hospital Medicine, Department of Medicine, University of California, San \\ Diego, La Jolla, CA, USA; ${ }^{5}$ Geriatric Research Education and Clinical Care Center, Tennessee Valley Healthcare System VA, Nashville, TN, USA; \\ ${ }^{6}$ Departments of Biomedical Informatics, Biostatistics, and Medicine, Vanderbilt University Medical Center, Nashville, TN, USA \\ Correspondence to: Arpan Patel, MD, PhD. Assistant Professor of Medicine, UCLA Division of Digestive Diseases, 1100 Glendon Avenue, Suite 850, \\ Room 810, Los Angeles, CA 90024, USA. Email: arpanpatel@mednet.ucla.edu. \\ Comment on: Garg SK, Goyal H, Obaitan I, Shah PA, et al. Incidence and predictors of 30-day hospital readmissions for liver cirrhosis: insights from \\ the United States National Readmissions Database. Ann Transl Med 2021;9:1052.
}

Submitted Oct 02, 2021. Accepted for publication Oct 14, 2021.

doi: $10.21037 / \mathrm{atm}-21-5270$

View this article at: https://dx.doi.org/10.21037/atm-21-5270

The burden of cirrhosis and cirrhosis-related deaths in the United States (US) has increased significantly over the past two decades, driven largely by climbing rates of alcohol use disorders and obesity (1). Over a similar timeframe, the number of hospitalizations has doubled and costs attributed to inpatient stays have tripled (2). This is even more striking among patients with cirrhosis, as the overall hospitalization trend in the United States slightly declined in the last decade (3). Readmissions are a prime example of low-value care, as they are associated with not only higher costs to payers but also greater psychosocial burden, reduced satisfaction, and increased mortality risk for patients (4). To address this rising healthcare burden, efforts to reduce readmissions in this population have emerged as an important priority for clinicians, health systems, and policymakers (5).

In a recent article, Garg and co-authors perform a nationwide analysis of readmissions for patients with cirrhosis with a focus on assessing modifiable risk factors that may be intervenable to reduce readmission risk (6). Using observational data of cirrhosis-related acute hospitalizations between 2010-2014 from the National Readmissions Database (NRD), this study demonstrated that $31.4 \%$ of patients with cirrhosis were readmitted within 30 days of hospitalization.
Orman and colleagues recently published a systematic review of studies reporting readmissions among patients with cirrhosis from 2000 to 2017 , finding a total of 21 retrospective studies (7). Their study provides a very nice reference for prior work, and the pooled estimate of 30-day readmissions was $26 \%$, aligning well with the current study. In addition, of the retrospective studies, twenty were singlecenter studies, highlighting the need for large, multi-center studies in this space.

Describing the context of readmissions is crucial because interventions and healthcare system changes to reduce readmissions should be tailored to the patient's comorbidities, reason for admission, and interventions during the index hospitalization (8). This can vary significantly by the primary reason for index hospitalization, with one study finding as much as $85 \%$ related readmissions for joint replacement (9) and another $33 \%$ for heart failure (10). Among hospitalized patients with cirrhosis, this can also vary significantly depending on the inclusion and exclusion criteria of the cohort, largely driven by the severity of liver disease. In the study by Garg and colleagues, diagnoses related to liver-related complications and substance use were found in $26 \%$ and $17 \%$ of readmissions in this cohort, respectively. In another study in an electronic health record study in the national VA, 60\% 
of admissions were related to cirrhosis related conditions (4). Garg and colleagues found that ascites and hepatic encephalopathy were the most common complications related to readmissions, findings that mirror those seen in other analyses of NRD and Department of Veterans Affairs (VA) $(4,11)$.

For readmissions that are potentially preventable, identifying root causes is a critical step. Potential causes should acknowledge not only patient factors, but also variables related to social determinants of health, patient function, and system-level performance (12). In this manuscript, Garg et al. identifies the following patient groups with the highest risk of readmissions: men, younger adults, Medicare and Medicaid beneficiaries, and individuals with comorbid conditions. Further analyses in this manuscript revealed that renal disease, chronic obstructive pulmonary disease, and diabetes mellitus were the most common conditions associated with readmissions. Viewing risk factors from a health system level, the authors also found that hospitalizations at metropolitan teaching hospitals carried the highest risk of readmissions; in particular, weekend admissions were associated with higher readmission risk while discharge to a nursing facility or short-term hospitals were protective.

As the authors suggested, understanding such predictors for readmissions can help inform development of risk prediction tools that can be used for quality improvement; however, this does come with caveats. First, when applied at the health system level, scores developed using national administrative data may have less favorable prediction utility, as shown in a study by Koola et al., where they portrayed this phenomenon occurring when prior tools were applied to the Veterans Affairs (VA) population (4). Though models developed utilizing administrative databases provide significantly more patient observations, thereby increasing modeling power and potentially increased generalizability, models derived from single institution and pauci-institution datasets containing granular clinical data such as laboratory tests, medication records, and social context variables may provide improved predictive performance.

Another important trend to note is the changing patient case-mix and outcome rates among patients with moderate to advanced liver disease. Orman and colleagues evaluated an Indiana statewide cohort of patients with a new diagnosis of cirrhosis and found a number of important trends. First, the rates of diagnosis were rising, the proportion of patients with alcoholic and nonalcoholic steatohepatitis were increasing, rates of viral hepatitis and hepatocellular carcinoma were declining, and overall mortality rates were declining (13). Davis and colleagues found among a national VA hospitalized population, across multiple outcomes, patient characteristics and healthcare delivery trends created information changes over time and led to risk modeling degradation (14). This highlights the need for continued evaluation of patients with cirrhosis in recent data to understand the ever-shifting trends and which characteristics that may be preventable could be good targets for intervention.

In addition, many studies evaluating cirrhosis are in the U.S. International Classification of Diseases (ICD)-9 era, including the study by Garg and colleagues. Since the switch to ICD-10, sustaining coding accuracy in both the inpatient and outpatient domains have been challenging, with a 2019 report showing only $\sim 60 \%$ accuracy for primary diagnoses and $\sim 40 \%$ for secondary diagnoses. Conditions such as opioid use disorders demonstrate an abrupt $14 \%$ increase in coding, whereas alcohol and tobacco dependence disorders saw a significant increase (15). Potential causes include that only $5 \%$ of ICD-10 codes have a 1 -to- 1 correspondence to semantically similar ICD-9 codes, a gradual learning curve as healthcare providers and coding/billing professionals acclimate to the ICD-10 system, and limitations to serial temporal assessments for data overlapping the ICD-9 to ICD-10 transition (15). This introduces further challenges and opportunities when using administrative data for population health tracking and interventions.

Understanding root causes can also be used to craft novel quality improvement and policy initiatives towards reducing readmissions. In general, such interventions should target high-risk groups identified in this study, as mentioned above. At the hospital level, ensuring the delivery of highquality cirrhosis care can be an effective intervention strategy. Tapper et al. reduced readmissions by $40 \%$ after applying a discharge checklist and electronic decision support tools (16). Similarly, presence of gastroenterology consultation during inpatient stays have been associated with reduced readmissions (17). The present study suggests that interventions that optimize care coordination and safe discharge plans may be an effective solution. In fact, several recent studies, including one randomized controlled trial, have highlighted the effect of specialty palliative care consultation in reducing readmissions for patients with cirrhosis, consistent with this concept (18-20). In the outpatient and community settings, only one intervention, incorporating multidisciplinary care management and same day hospitals for procedures in hepatology clinics, 
effectively reduced both readmissions and mortality in a cohort of patients with cirrhosis (21).

In the manuscript, Garg and co-authors call for policylevel interventions to help with reducing readmissions, similar to how the Hospital Readmissions Reduction Program (HRRP) has been implemented for comparable serious illnesses such as congestive heart failure (CHF) (22). However, endorsing such solutions for readmissions may not lead to desired outcomes. For instance, though HRRP has been associated with reduced readmissions for CHF, 1-year mortality increased over the timeframe of implementation (23). Interestingly, HRRP was not associated with reduced rates of readmissions in patients who had a comorbid diagnosis of cirrhosis (24).

The problem of addressing readmissions in patients with cirrhosis is at a critical crossroads. Garg and authors offer some unique insights that further understanding of high-risk populations and the types of solutions that can be offered to them. However, with the mounting burden of illness and healthcare utilization, the time to act is now. Clinicians and health systems must invest in developing and testing tools that predict readmission risk for this population within their health systems, ideally capturing a more granular context of readmissions. In tandem, planned interventions, including those that support care coordination and high quality cirrhosis care in the inpatient and outpatient settings, should be tested. Policymakers should consider supporting such interventions, recognizing that Medicare and Medicaid beneficiaries are at higher risk of readmissions. As described in Garg et al. and similar studies, we have made significant strides over the past decade in understanding readmissions for patients with cirrhosis. In the next decade, let us strive to act on these changes to finally meet the needs of this vulnerable population and their communities.

\section{Acknowledgments}

Funding: None.

\section{Footnote}

Provenance and Peer Review: This article was commissioned by the editorial office, Annals of Translational Medicine. The article did not undergo external peer review.

Conflicts of Interest: All authors have completed the ICMJE uniform disclosure form (available at http://dx.doi. org/10.21037/atm-21-5270). The authors have no conflicts of interest to declare.

Ethical Statement: The authors are accountable for all aspects of the work in ensuring that questions related to the accuracy or integrity of any part of the work are appropriately investigated and resolved.

Open Access Statement: This is an Open Access article distributed in accordance with the Creative Commons Attribution-NonCommercial-NoDerivs 4.0 International License (CC BY-NC-ND 4.0), which permits the noncommercial replication and distribution of the article with the strict proviso that no changes or edits are made and the original work is properly cited (including links to both the formal publication through the relevant DOI and the license). See: https://creativecommons.org/licenses/by-nc-nd/4.0/.

\section{References}

1. Tapper EB, Parikh ND. Mortality due to cirrhosis and liver cancer in the United States, 1999-2016: observational study. BMJ 2018;362:k2817.

2. Zou B, Yeo YH, Jeong D, et al. A Nationwide Study of Inpatient Admissions, Mortality, and Costs for Patients with Cirrhosis from 2005 to 2015 in the USA. Dig Dis Sci 2020;65:1520-8.

3. HCUP Fast Stats. Healthcare Cost and Utilization Project (HCUP). April Agency for Healthcare Research and Quality R, MD. Available online: www.hcup-us.ahrq.gov/ faststats/national/inpatienttrends.jsp. Accessed September 29, 2021.

4. Koola JD, Ho SB, Cao A, et al. Predicting 30-Day Hospital Readmission Risk in a National Cohort of Patients with Cirrhosis. Dig Dis Sci 2020;65:1003-31.

5. Tapper EB, Volk M. Strategies to Reduce 30Day Readmissions in Patients with Cirrhosis. Curr Gastroenterol Rep 2017;19:1.

6. Garg SK, Goyal H, Obaitan I, et al. Incidence and predictors of 30-day hospital readmissions for liver cirrhosis: insights from the United States National Readmissions Database. Ann Transl Med 2021;9:1052.

7. Orman ES, Ghabril M, Emmett TW, et al. Hospital Readmissions in Patients with Cirrhosis: A Systematic Review. J Hosp Med 2018. [Epub ahead of print].

8. Benbassat J, Taragin M. Hospital readmissions as a measure of quality of health care: advantages and limitations. Arch Intern Med 2000;160:1074-81.

9. Khouri RK Jr, Hou H, Dhir A, et al. What is the impact 


\section{Page 4 of 4}

of a clinically related readmission measure on the assessment of hospital performance? BMC Health Serv Res 2017;17:781.

10. Reddy YNV, Borlaug BA. Readmissions in Heart Failure: It's More Than Just the Medicine. Mayo Clin Proc 2019;94:1919-21.

11. Mumtaz K, Issak A, Porter K, et al. Validation of Risk Score in Predicting Early Readmissions in Decompensated Cirrhotic Patients: A Model Based on the Administrative Database. Hepatology 2019;70:630-9.

12. Kansagara D, Englander H, Salanitro A, et al. Risk prediction models for hospital readmission: a systematic review. JAMA 2011;306:1688-98.

13. Orman ES, Roberts A, Ghabril M, et al. Trends in Characteristics, Mortality, and Other Outcomes of Patients With Newly Diagnosed Cirrhosis. JAMA Netw Open 2019;2:e196412.

14. Davis SE, Lasko TA, Chen G, et al. Calibration drift in regression and machine learning models for acute kidney injury. J Am Med Inform Assoc 2017;24:1052-61.

15. Khera R, Dorsey KB, Krumholz HM. Transition to the ICD-10 in the United States: An Emerging Data Chasm. JAMA 2018;320:133-4.

16. Tapper EB, Finkelstein D, Mittleman MA, et al. A Quality Improvement Initiative Reduces 30-Day Rate of Readmission for Patients With Cirrhosis. Clin Gastroenterol Hepatol 2016;14:753-9.

17. Serper M, Kaplan DE, Lin M, et al. Inpatient Gastroenterology Consultation and Outcomes of

Cite this article as: Patel A, Koola JD, Matheny ME. Reducing readmissions in patients with cirrhosis: the time to act is now. Ann Transl Med 2021;9(22):1637. doi: 10.21037/atm-21-5270

\section{Patel et al. Reducing readmissions in patients with cirrhosis}

Cirrhosis-Related Hospitalizations in Two Large National Cohorts. Dig Dis Sci 2021. [Epub ahead of print].

18. Adejumo AC, Kim D, Iqbal U, et al. Suboptimal Use of Inpatient Palliative Care Consultation May Lead to Higher Readmissions and Costs in End-Stage Liver Disease. J Palliat Med 2020;23:97-106.

19. Shinall MC Jr, Karlekar M, Martin S, et al. COMPASS: A Pilot Trial of an Early Palliative Care Intervention for Patients With End-Stage Liver Disease. J Pain Symptom Manage 2019;58:614-622.e3.

20. Rush B, Fruhstofer C, Walley KR, et al. Palliative medicine and hospital readmissions in end-stage liver disease. BMJ Support Palliat Care 2019. [Epub ahead of print].

21. Morando F, Maresio G, Piano S, et al. How to improve care in outpatients with cirrhosis and ascites: a new model of care coordination by consultant hepatologists. J Hepatol 2013;59:257-64.

22. McIlvennan CK, Eapen ZJ, Allen LA. Hospital readmissions reduction program. Circulation 2015;131:1796-803.

23. Gupta A, Allen LA, Bhatt DL, et al. Association of the Hospital Readmissions Reduction Program Implementation With Readmission and Mortality Outcomes in Heart Failure. JAMA Cardiol 2018;3:44-53.

24. Rosenblatt R, Cohen-Mekelburg S, Shen N, et al. Cirrhosis as a Comorbidity in Conditions Subject to the Hospital Readmissions Reduction Program. Am J Gastroenterol 2019;114:1488-95. 